\title{
Preservación de reborde en el sector posterior: Una revisión sistemática
}

\author{
Ridge preservation in posterior sites: A systematic review. \\ Edwin Ramos-Pilco ${ }^{1}$, Gustavo Allasi Tejada ${ }^{1}$, Marco Alarcón Palacios ${ }^{1,2}$ \\ RESUMEN
}

Objetivo: Evaluar mediante una revisión sistemática los cambios dimensionales producidos después de una extracción dental en el sector posterior empleando técnicas de preservación de reborde alveolar y cicatrización convencional. Material y métodos: Dos revisores independientes y calibrados realizaron una búsqueda electrónica de ensayos clínicos aleatorizados publicados hasta diciembre del 2018 en Pubmed y Cochrane la cual fue complementada con una búsqueda manual en las revistas de mayor impacto en Periodoncia e Implantología según el ISI Web of Science. La evaluación del riesgo de sesgo en los estudios incluidos fue realizada siguiendo el manual de Cochrane para intervenciones de revisiones sistemáticas Versión 5.1.0. Resultados: Un total de 435 piezas posteriores, entre premolares y molares, fueron evaluadas en los estudios incluidos a partir de los 3 meses post extracción. Se obtuvo valores estadísticamente significativos para el grupo que realizó la preservación de reborde (pérdida ósea vertical desde $-0,25$ hasta $-1,53 \mathrm{~mm}$ y a nivel horizontal desde $-0,91$ hasta $-2,87$ mm) en comparación al grupo control (pérdida ósea vertical desde -0,71 hasta -3,1 mm y a nivel horizontal desde -2,26 hasta -3,96 mm). El biomaterial más utilizado fue el xenoinjerto más membrana de colágeno. Los estudios incluidos manifestaron un bajo riesgo de sesgo. Conclusiones: La preservación de reborde en sitios posteriores es recomendada porque conduce a mantener los tejidos duros y blandos, reduce la neumatización sinusal y minimiza la reabsorción del hueso crestal simplificando así los procedimientos para una buena posición tridimensional del implante.

PALABRAS CLAVE: Implantes dentales; extracción dental; diente molar; proceso alveolar; reabsorción ósea; revisión sistemática.

Segunda Especialidad en Periodoncia e Implantología, Facultad de Ciencias de la Salud, Universidad Privada de Tacna. Tacna, Perú. Departamento Académico de Clínica Estomatológica, Facultad de Estomatología, Universidad Peruana Cayetano Heredia. Lima, Perú. 


\section{SUMMARY}

Objective: To evaluate the dimensional changes produced after a dental extraction in the posterior sites using techniques of preservation of ridge and conventional healing. Materials and methods: Two independent and calibrated reviewers conducted an electronic search of randomized clinical trials published until December 2018 in Pubmed and Cochrane, which was complemented by a manual search in the journals with the greatest impact in Periodontics and Implantology according to the ISI Web of Science. The evaluation of the risk of bias in the included studies was carried out following the Cochrane manual for interventions of systematic reviews Version 5.1.0. Results: A total of 435 posterior pieces, between premolars and molars, were evaluated in the included studies after 3 months post extraction. Statistically significant values were obtained for the group that performed ridge preservation (vertical bone loss from -0.25 to $-1.53 \mathrm{~mm}$ and horizontally from -0.91 to $-2.87 \mathrm{~mm}$ ) compared to the control group (vertical bone loss from -0.71 to $-3.1 \mathrm{~mm}$ and at horizontal level from -2.26 to $-3.96 \mathrm{~mm}$ ). The most used biomaterial was the xenograft plus collagen membrane. The included studies showed a low risk of bias. Conclusions: The ridge preservation in posterior sites leads to: maintaining hard and soft tissues, reduces sinus pneumatization and minimizes the resorption of the crestal bone, generating a great volume of bone tissue, thus simplifying the procedures for a good three-dimensional position of the implant.

\section{KEYWORDS: Dental implants; tooth extraction; molar; alveolar process; bone resorption; systematic review.}

\section{INTRODUCCIÓN}

Después de una extracción dental, existe una pérdida ósea horizontal hasta los 6 meses de 3,79 mm (2,46$4,56 \mathrm{~mm} ; 29-63 \%)$ y vertical de $1,24 \mathrm{~mm}(0,8-1,5$ $\mathrm{mm} ; 11-22 \%)$, siguiendo una reducción gradual a partir de entonces a lo largo de la vida $(1,2)$. Está demostrado que existe una mayor reabsorción de la tabla vestibular que la lingual $(3,4)$.

Estas deficiencias óseas y a la vez de tejidos blandos en la zona implantar son hallazgos clínicos comunes, su presencia puede llevar a una mala posición tridimensional del implante complicando la rehabilitación protésica, un aumento en la pérdida ósea crestal e inflamación de los tejidos blandos (mucositis, periimplantitis) $(5,6)$.

Una terapia con gran aceptación es la colocación de implantes inmediatos en alveolos post extractivos, pero los resultados clínicos estudiados en dientes posteriores parecen no ser tan exactos como en sitios anteriores debido a razones morfológicas y anatómicas, es por ello que la preservación del reborde surge como una alternativa para la rehabilitación implantosoportada en molares y premolares (7).

Se define el término de "preservación de reborde" (PRA) como preservar el volumen del reborde dentro del lecho ya existente en el momento de la extracción $(8,9,10)$, por lo tanto, las razones para emplear esta técnica son: mantener un volumen de reborde estable para optimizar los resultados funcionales, estéticos y simplificar los tratamientos posteriores (11).

La mayoría de estudios se enfocan en la preservación de reborde del sector anterior, ignorando la región posterior de la maxila y mandíbula, donde se localiza el seno maxilar y trayecto del nervio dentario inferior respectivamente, limitando la colocación de implantes $(4,9)$.

El objetivo del presente estudio fue evaluar los cambios dimensionales producidos después de una extracción dental en el sector posterior empleando técnicas de PRA y cicatrización convencional.

\section{MATERIAL Y MÉTODOS}

\section{Protocolo y pregunta de investigación}

Un protocolo fue desarrollado a priori, con el propósito de responder la siguiente pregunta: ¿Es necesaria la preservación de reborde alveolar, para la futura colocación de implantes en el sector posterior? Este protocolo cumplió las recomendaciones del reporte para revisiones sistemáticas PRISMA (Preferred Reporting Items for Systematic Review and MetaAnalyses) (12). 


\section{Criterios de inclusión (PICOS)}

- Población: Dientes posteriores (premolares y molares) sin periodontitis crónica que necesitan extracción en pacientes sin compromiso sistémico.

- Intervención: Colocación de algún biomaterial.

- Comparación: Cicatrización convencional.

- Resultados: Comparación clínica y/o tomográfica a partir de 3 meses.

- Diseño de estudio: Ensayos clínicos aleatorizados (ECAs).

\section{Criterios de exclusión}

Estudios que no presentan grupo control, dientes anteriores, pacientes con hábitos nocivos (fumadores, drogas), embarazo, lactancia y pacientes menores 18 años.

\section{Tipos de resultados}

El objetivo primario de esta revisión sistemática fue evaluar los cambios tomográficos y clínicos de la pérdida ósea vertical y horizontal a partir de los 3 meses, medida en milímetros. El objetivo secundario fue evaluar el riesgo de sesgo de los ensayos clínicos sobre preservación de reborde en sitios posteriores.

\section{Búsqueda electrónica y manual}

Se realizó una búsqueda exhaustiva en la base de datos MEDLINE (PubMed) y en la biblioteca Cochrane, hasta diciembre 2018. La estrategia de búsqueda utilizó los siguientes términos: (ridge augmentation) OR ridge preservation) OR socket preservation) OR alveolar ridge) OR alveolar ridge preservation)) AND (molar extraction) OR posterior sites) OR posterior maxillary) OR posterior maxilla) OR posterior implants) OR posterior alveolar bone) OR posterior maxillae) OR posterior regions) OR posterior teeth), adicionalmente se examinaron las listas de referencia de cualquier artículo potencial. Las siguientes revistas fueron buscadas manualmente hasta diciembre 2018, por ser las de mayor impacto en Periodoncia e Implantología oral: Journal of Clinical Periodontology, Journal of Periodontology, Clinical Oral Implants Research, International Journal of Oral \& Maxillofacial Implants, European Journal of Oral Implantology, Implant Dentistry, International Journal of Periodontics and Restorative Dentistry,
International Journal of Prosthodontics, Journal of Prosthetic Dentistry and Clinical Implant Dentistry and Related Research. Asimismo, se complementó esta etapa realizándose la búsqueda en la literatura gris: www.opengrey.eu.

\section{Selección de artículos y extracción de datos}

Dos revisores independientes (E. R. y G. A.) examinaron los títulos, resumen y textos completos de los artículos que fueron identificados y extrajeron los datos. Si existió desacuerdo entre los revisores se resolvió mediante una discusión hasta que se alcanzó un consenso. Cuando no se llegó a un consenso un tercer revisor (M.A.) fue consultado. Para calibrar la confiabilidad entre los revisores, se calcularon las concordancias mediante el coeficiente Kappa (K: $0.8)$.

\section{Evaluación de la calidad}

Se realizó el riesgo de sesgo de los ECAs incluidos siguiendo el manual de Cochrane para intervenciones de revisiones sistemáticas Versión 5.1.0. (13). Se evaluaron seis criterios principales de calidad: generación de la secuencia, ocultamiento de la información, cegamiento de los participantes y del personal, cegamiento de los evaluadores de los resultados, datos de resultados incompletos, reporte selectivo y otros sesgos. Estos criterios se calificaron como bajo (verde), no claro (amarillo) y alto riesgo de sesgo (rojo), según la descripción dada para cada campo individual.

\section{RESULTADOS}

Un diagrama de flujo de los resultados se muestra en la figura 1. La búsqueda de la literatura, realizada por dos revisores independientes (E.R. y G.A.), identificó un total de 1679 potenciales artículos, de los cuales 1580 fueron excluidos después de la evaluación de títulos y resúmenes, a continuación, se revisaron el texto completo de los 19 artículos potenciales. Finalmente, un total de 09 estudios cumplieron los criterios de inclusión y fueron evaluados en la presente revisión sistemática. Los resultados del riesgo de sesgo de los ECAs se encuentran en la tabla 1. Siguiendo las recomendaciones de Higgins y Green (13), siete ensayos clínicos fueron de bajo riesgo de sesgo $(9,14,15,16,17,18,19)$ destacando 
Figura 1. Diagrama de flujo (formato PRISMA) filtración y proceso de selección
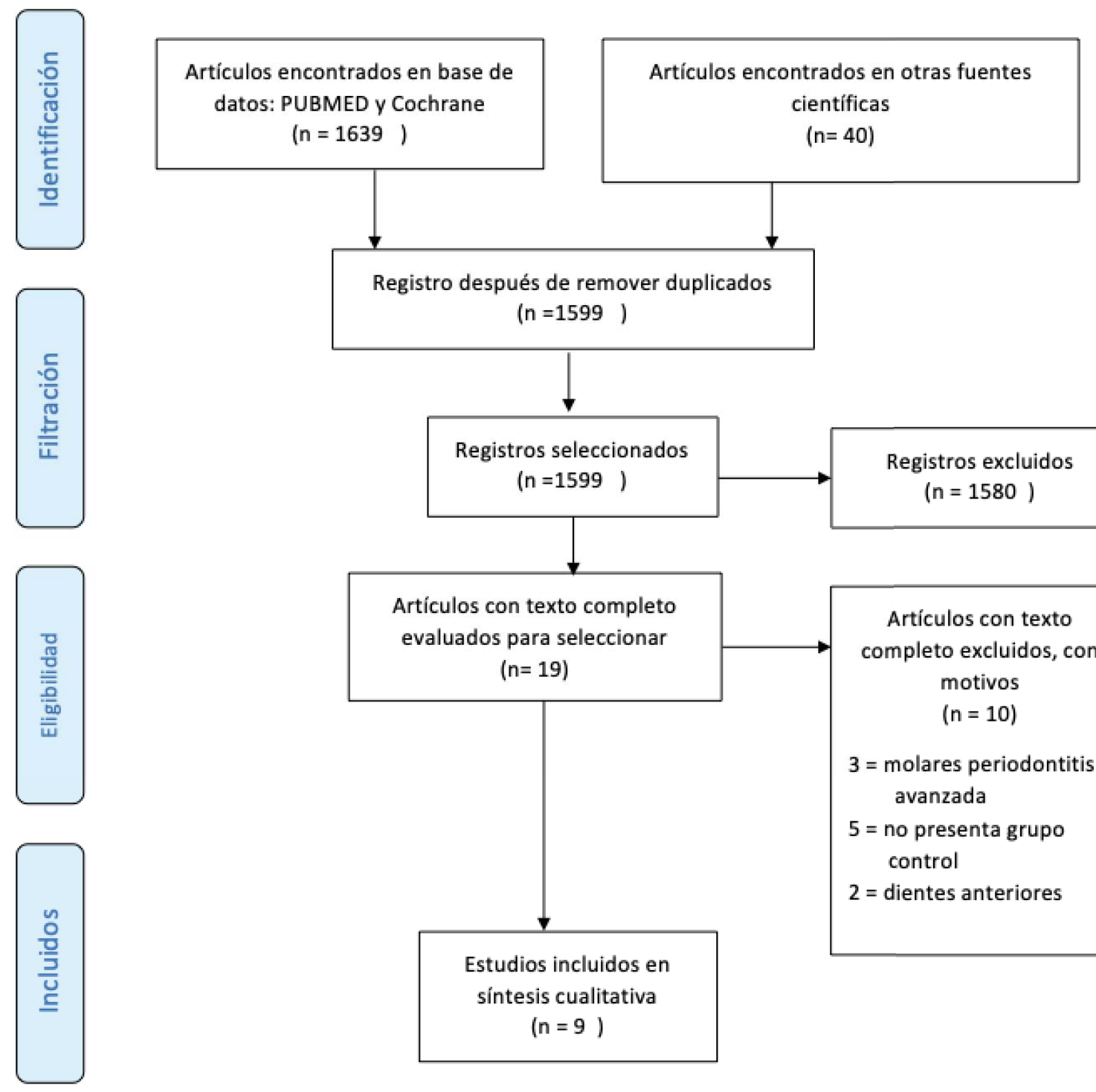

Registros excluidos $(n=1580)$
Artículos con texto completo excluidos, con motivos $(n=10)$

$3=$ molares periodontitis avanzada $5=$ no presenta grupo control

$2=$ dientes anteriores que el criterio "datos de resultados incompletos" fue cumplido por todos los estudios mientras que el ítem que menos fue cumplido fue el del "cegamiento de pacientes y operadores”.

Fueron intervenidas un total de 435 piezas posteriores entre premolares y molares. El biomaterial usado en 8 artículos fue el xenoinjerto más membrana de colágeno (MC) y solo Walker et al., utilizaron el aloinjerto más politetrafluoroetileno denso (PTFEd) (16). Cinco estudios emplearon el cierre de la herida por primera intención con un colgajo desplazado a coronal $(14,17,19,20,21)$ y cuatro por segunda intención, dejando expuesta la membrana de colágeno al medio bucal y estabilizado con suturas $(9,15,16,18)$. Cuatro ECAs utilizaron el método clínico para evaluar los cambios dimensionales que consiste en elevar un colgajo durante la extracción y otra durante la reevaluación $(14,15,18,19)$. Cinco ECAs usaron el método imagenológico que incluye la utilización de radiografías panorámicas y tomografía computarizada de haz cónico (TCHC) $(9,16,17,20,21)$. Se obtuvieron cambios dimensionales a nivel vertical vestibular medial desde $-0,25$ hasta $-1,53 \mathrm{~mm}$ y a nivel horizontal desde $-0,91$ hasta $-2,87 \mathrm{~mm}$, en el grupo control a nivel vertical vestibular medial desde $-0,71$ hasta $-3,1 \mathrm{~mm}$ y a nivel horizontal desde $-2,26$ hasta $-3,96 \mathrm{~mm}$, encontrándose diferencias 
Tabla 1. Formato PICO (P: población, I: intervención, C: comparación, O: resultados)

\begin{tabular}{|c|c|c|c|c|c|c|c|}
\hline AUTOR & DISEÑO & POBLACIÓN & INTERVENCIÓN & COMPARACIÓN & \multicolumn{2}{|l|}{ RESULTADOS } & SEGUIMIENTO \\
\hline $\begin{array}{l}\text { Jung R. } \\
2018\end{array}$ & $\begin{array}{l}\text { ECA; boca } \\
\text { dividida }\end{array}$ & $\begin{array}{l}\text { E: } 18 \text { Pm y M } \\
\text { C: }\end{array}$ & $\begin{array}{l}\text { ROG: xenoinjerto } \\
+ \text { MC } \\
\text { (Membrana } \\
\text { expuesta) }\end{array}$ & $\begin{array}{l}\text { Cicatrización } \\
\text { convencional }\end{array}$ & \multicolumn{2}{|c|}{$\begin{array}{l}\text { TCHC; } \quad \text { P }<0,05 \\
A V . \quad \mathbf{E}:-0,25 \mathrm{~mm} ; \mathbf{C}:-1,25 \mathrm{~mm} \\
A P . \quad \mathbf{E}:-0,26 \mathrm{~mm} ; \mathbf{C}:-0,89 \mathrm{~mm} \\
\text { H-1. } \mathbf{E}:-1,33 \mathrm{~mm} ; \mathbf{C}:-2,78 \mathrm{~mm}\end{array}$} & 6 meses \\
\hline $\begin{array}{l}\text { Levi I. } \\
2017\end{array}$ & $\begin{array}{l}\text { ECA; } \\
\text { grupos } \\
\text { paralelos }\end{array}$ & $\begin{array}{l}\text { E: } 21 \text { Pms y Ms } \\
\text { C: } 42 \text { Pms y Ms }\end{array}$ & $\begin{array}{l}\text { ROG: xenoninjerto } \\
\text { + CDC }\end{array}$ & $\begin{array}{l}\text { Cicatrización } \\
\text { convencional }\end{array}$ & \multicolumn{2}{|c|}{$\begin{array}{l}\text { Rx panorámica } p=0,0019 \\
\text { Diferencia (A-piso seno): } \\
\text { E: }-0,32(0,09) \mathrm{mm} \\
\text { C: }-1,26(0,28) \mathrm{mm}\end{array}$} & 12 meses \\
\hline $\begin{array}{l}\text { Guarnieri } \\
\text { R. } 2017\end{array}$ & $\begin{array}{l}\text { ECA; } \\
\text { grupos } \\
\text { paralelos }\end{array}$ & $\begin{array}{l}\text { E: a) } 09 \text { Pms y Ms }{ }^{m} \\
\text { b) } 08 \text { Pms y Ms }{ }^{g m} \\
\text { C: } 09 \text { Pms y Ms }\end{array}$ & $\begin{array}{l}\text { a) solo MC } \\
\text { b) xenoinjerto + } \\
\text { MC } \\
\text { (membrana } \\
\text { expuesta) }\end{array}$ & $\begin{array}{l}\text { Cicatrización } \\
\text { convencional }\end{array}$ & \multicolumn{2}{|c|}{$\begin{array}{l}\text { Clínico } \quad \mathrm{p}<0,001 \\
\mathrm{~A}^{\mathrm{m}}:-0,55(0,11) \mathrm{mm}, \mathrm{A}^{\mathrm{gm}}:-0,37 \\
(0,7) \mathrm{mm} \\
\mathrm{H}^{\mathrm{m}}:-1,21(0,69) \mathrm{mm}, \mathrm{H}^{\mathrm{gm}}:-0,91 \\
(0,53) \mathrm{mm} \\
\mathrm{A} ; \mathrm{C}:-2,09(0,19) \mathrm{mm} \\
\mathrm{H} ; \text { C: }-3,96(0,87) \mathrm{mm}\end{array}$} & 3 meses \\
\hline $\begin{array}{l}\text { Barone A. } \\
2016\end{array}$ & $\begin{array}{l}\text { ECA; } \\
\text { grupos } \\
\text { paralelos }\end{array}$ & $\begin{array}{l}\text { E: a) } 30 \text { Pms y Ms coll } \\
\text { b) } 30 \text { Pms y Ms cort } \\
\text { C: } 30 \text { Pms y Ms }\end{array}$ & $\begin{array}{l}\text { a) xenoinjerto + } \\
\text { MC } \\
\text { b) xenoinjerto + } \\
\text { MC } \\
\text { (membrana } \\
\text { expuesta) }\end{array}$ & $\begin{array}{l}\text { Cicatrización } \\
\text { convencional }\end{array}$ & \multicolumn{2}{|c|}{$\begin{array}{l}\text { Clínico } \quad \mathrm{p}<0,0001 \\
\mathrm{~A}^{\text {colll }}:-0,57 \mathrm{~mm}, \mathrm{~A}^{\text {cort: }}-0,30 \mathrm{~mm} \\
\mathrm{H}^{\text {coll: }}:-0,93 \mathrm{~mm}, \mathrm{H}^{\text {cort: }}-1,33 \mathrm{~mm} \\
\text { A; C: }-2,10 \mathrm{~mm} \\
\text { H; C: }-3,60 \mathrm{~mm}\end{array}$} & 3 meses \\
\hline $\begin{array}{l}\text { Pang C. } \\
2016\end{array}$ & $\begin{array}{l}\text { ECA; } \\
\text { grupos } \\
\text { paralelos }\end{array}$ & $\begin{array}{l}\text { E: } 30 \text { PPM } \\
\text { C: } 30 \text { PPM }\end{array}$ & $\begin{array}{l}\text { ROG: xenoinjerto } \\
+ \text { MC + CDC }\end{array}$ & $\begin{array}{l}\text { Cicatrización } \\
\text { convencional }\end{array}$ & \multicolumn{2}{|c|}{$\begin{array}{l}\text { TCHC; } \quad \mathrm{p}<0,05 \\
\text { Nivel } A^{a} \text { : } \\
\text { A; E: }-1,53(0,26) \mathrm{mm} \text {; C: }-2,92 \\
(0,31) \mathrm{mm} \\
\text { H; E: }-2,87(0,25) \mathrm{mm} \text {; C: }-3,26 \\
(0,44) \mathrm{mm} \\
\text { Nivel } A^{b}: \\
\text { A; E: }-2,48(0,22) \mathrm{mm} \text {; C: }-3,17 \\
(0,37) \mathrm{mm} \\
\text { H; E: }-3,05(0,18) \mathrm{mm} \text {; C: }-3,82 \\
(0,33) \mathrm{mm}\end{array}$} & 6 meses \\
\hline $\begin{array}{l}\text { Walker C. } \\
2016\end{array}$ & $\begin{array}{l}\text { ECA; } \\
\text { grupos } \\
\text { paralelos }\end{array}$ & $\begin{array}{l}\mathrm{E}: 20 \mathrm{Ms} \\
\mathrm{C}: 20 \mathrm{Ms}\end{array}$ & $\begin{array}{l}\text { ROG: Aloinjerto + } \\
\text { PTFEd } \\
\text { (membrana } \\
\text { expuesta) }\end{array}$ & $\begin{array}{l}\text { Cicatrización } \\
\text { convencional }\end{array}$ & \multicolumn{2}{|c|}{$\begin{array}{l}\text { TCHC: } \quad p=.01 \\
\text { A; E: }-1,12(1,60) \mathrm{mm} ; \mathrm{C}:-2,60 \\
(2,06) \mathrm{mm} \\
\quad \mathrm{P}=.34 \\
\text { H; E: }-2,48(2,86) \mathrm{mm} \text {; C: }-3,11 \\
(3,83) \mathrm{mm}\end{array}$} & 3 meses \\
\hline $\begin{array}{l}\text { Lei C. } \\
2015\end{array}$ & $\begin{array}{l}\text { ECA; boca } \\
\text { dividida }\end{array}$ & $\begin{array}{l}\text { E: } 16 \text { Pm y M } \\
\text { C: }\end{array}$ & $\begin{array}{l}\text { ROG: Xenoinjerto } \\
\text { + MC + CDC }\end{array}$ & $\begin{array}{l}\text { Cicatrización } \\
\text { convencional }\end{array}$ & \multicolumn{2}{|c|}{$\begin{array}{l}\text { TCHC; } \quad \text { p }<0,05 \\
\text { A; E: }-0,55(1,05) \mathrm{mm} ; \mathbf{C}:-0,71 \\
(1,41) \mathrm{mm} \\
\text { H; E: }-0,99(0,94) \mathrm{mm} ; \mathbf{C}:-2,26 \\
(1,29) \mathrm{mm}\end{array}$} & 6 meses \\
\hline $\begin{array}{l}\text { Barone A. } \\
2012\end{array}$ & $\begin{array}{l}\text { ECA; } \\
\text { grupos } \\
\text { paralelos }\end{array}$ & $\begin{array}{l}\text { E: } 29 \text { Pms y Ms } \\
\text { C: } 29 \text { Pms y Ms }\end{array}$ & $\begin{array}{l}\text { ROG: Xenoinjerto } \\
+ \text { MC } \\
\text { (membrana } \\
\text { expuesta) }\end{array}$ & $\begin{array}{l}\text { Cicatrización } \\
\text { convencional }\end{array}$ & \multicolumn{2}{|c|}{$\begin{array}{l}\text { Guías de acrílico } \\
\text { A; E: -1,1 }(0,96) \mathrm{mm} \text {; C: -2,1 } \\
(0,6) \mathrm{mm} \\
\text { H; E: -1,6 }(0,55) \mathrm{mm} ; \mathbf{C}:-3,6 \\
(0,72) \mathrm{mm}\end{array}$} & 4 meses \\
\hline $\begin{array}{l}\text { Festa V. } \\
2011\end{array}$ & $\begin{array}{l}\text { ECA; boca } \\
\text { dividida }\end{array}$ & $\begin{array}{l}\text { E: } 15 \text { Pms } \\
\text { C: }\end{array}$ & $\begin{array}{l}\text { ROG: Xenoinjerto } \\
+ \text { MC } \\
+ \text { CDC }\end{array}$ & $\begin{array}{l}\text { Cicatrización } \\
\text { convencional }\end{array}$ & 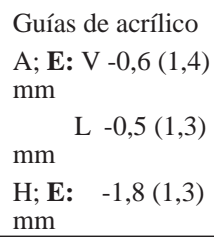 & $\begin{array}{l}\mathrm{P}<0,05 \\
\mathrm{C}: \mathrm{V}-3,1 \\
(1,3) \mathrm{mm} \\
\mathrm{L}-2,4 \\
(1,6) \mathrm{mm} \\
\mathrm{C}: \quad-3,7 \\
(1,2) \mathrm{mm}\end{array}$ & 6 meses \\
\hline
\end{tabular}

ECA: Ensayo clínico aleatorizado; C: control; E: experimental; MC: membrana colágeno; TCHC: Tomografía computarizada de haz cónico; AV: altura tabla vestibular; AP: altura tabla palatina; H-1: ancho del alveolo a 1mm tabla lingual; CDC: colgajo desplazado coronal; MV: medida vertical de cresta ósea al piso de seno; PPM: piezas posteriores mandibulares; Pm: premolares; Pms: premolares superiores; M: molares; Ms: molares superiores; A: altura reborde alveolar; H: ancho reborde alveolar

; ${ }^{\text {a: }}$ defecto vestibular de 3-5 mm; b: defecto vestibular > 5mm; An: anterior; Po: posterior; V: vestibular; L: lingual; Rx: radiografía; EOD: escáner óptico dimensional

; ' : grupo usa solo MC; ${ }^{\text {gm: }}$ hueso bovino + MC; PTFEd: Membrana politetrafluoroetileno denso. 
estadísticamente significativas a favor del grupo de preservación de reborde versus el grupo de cicatrización convencional.

\section{DISCUSIÓN}

\section{Calidad de la evidencia y sesgos potenciales en el proceso de revisión}

De acuerdo a las definiciones de Higgins y Green (13), la presente revisión sistemática revela un bajo riesgo de sesgo en la mayoría de los estudios incluidos (tabla 2), lo cual resalta que la metodología usada en la mayoría de ECAs son reproducibles y tienen bastante confiabilidad para la aplicación clínica en la toma de decisiones en los pacientes. Cabe mencionar la variabilidad en la medición de los resultados al utilizarse métodos clínicos e imagenológicos. En cuanto a las mediciones imagenológicas se demostró que la tomografía es más precisa en comparación con los métodos radiográficos bidimensionales como la radiografía panorámica siendo la media de distorsión expresada en porcentajes de 1,8\% para TCHC y $23,5 \%$ para radiografías panorámicas $(22,23)$. Asimismo, Bassir et al., demostraron que las medidas radiográficas en la técnica PRA pueden no reflejar las verdaderas dimensiones de la cresta, ya que no es posible diferenciar la formación de hueso nuevo de las partículas de injerto restantes, por lo tanto, los resultados de los análisis imagenológicos deben interpretarse con cautela (2). A pesar de estas diferencias, los resultados de todos los estudios muestran una reducción mínima del reborde para los grupos que emplearon biomaterial en los alveolos post extracción, tanto en los que utilizaron medidas clínicas, así como las imagenológicas.

\section{Hallazgos comparados con otras revisiones}

Los resultados de la presente revisión sistemática son similares a las estudios que evalúan la preservación de reborde en dientes anteriores (24) y a estudios que incluyen dientes anteriores $\mathrm{y}$ posteriores $(2,6,11,25,26,27,28)$, donde se pone de manifiesto el beneficio potencial de las terapias de preservación de reborde en comparación a la cicatrización convencional, también es necesario recalcar que estos procedimientos no evitan los cambios dimensionales del tejido óseo después de la extracción dental. En el informe de consenso de la Osteology Foundation (4), llegaron a la conclusión que los sitios molares en comparación de las piezas anteriores presentan

Tabla 2. Resumen de riesgo de sesgo (13)

\begin{tabular}{|c|c|c|c|c|c|c|}
\hline AUTOR & $\begin{array}{l}\text { GENERACIÓN } \\
\text { SECUENCIA }\end{array}$ & $\begin{array}{l}\text { OCULTAMIENTO } \\
\text { INFORMACIÓN }\end{array}$ & $\begin{array}{l}\text { CEGAMIENTO } \\
\text { PACIENTES Y } \\
\text { OPERADORES }\end{array}$ & $\begin{array}{c}\text { CEGAMIENTO } \\
\text { DE LOS } \\
\text { EVALUADORES } \\
\text { DE RESULTADOS }\end{array}$ & $\begin{array}{c}\text { DATOS } \\
\text { RESULTADOS } \\
\text { INCOMPLETOS }\end{array}$ & $\begin{array}{c}\text { NOTIFICACIÓN } \\
\text { SELECTIVA } \\
\text { RESULTADOS }\end{array}$ \\
\hline Jung R. 2018 & & & & & & \\
\hline Levi I. 2017 & & & & & & \\
\hline $\begin{array}{l}\text { Guarnieri R. } \\
2017\end{array}$ & & & & & & \\
\hline Barone A. 2016 & & & & & & \\
\hline Pang C. 2016 & & & & & & \\
\hline Walker C. 2016 & & & & & & \\
\hline Lei C. 2015 & & & & & & \\
\hline Barone A. 2012 & & & & & & \\
\hline Festa V. 2011 & & & & & & \\
\hline
\end{tabular}


limitadas indicaciones para la colocación de implantes inmediatos tipo 1 debido a razones anatómicas $(25,29)$ y morfológicas, parece que los sitios posteriores muestran un patrón diferente de remodelación ósea después de la extracción dental, considerando la altura de la tabla vestibular, existe mayor reabsorción en los sitios posteriores comparados con los sitios anteriores $(30,31)$, especialmente en el maxilar superior donde una relación cercana del ápice de la raíz al piso sinusal, puede llevar a una neumatización del seno maxilar (32) y más la pérdida de hueso crestal, trae como resultado la disminución drástica de la dimensión vertical de este sitio (39) y en la mandíbula la pérdida de la pieza dental puede comprometer la colocación del implante por la cercanía del nervio dentario inferior. La regeneración ósea guiada (ROG) es el método común para resolver este problema, generalmente hay dos opciones para incrementar la altura del reborde en las zonas posteriores: elevación de piso de seno maxilar y regeneración ósea vertical, sin embargo, existen tres problemas principales asociados con el aumento óseo: costo y duración del tratamiento, la alta morbilidad del paciente y no necesariamente tasas de éxito ideales $(33,34)$. Por este motivo por los resultados encontrados en la presente revisión es necesaria aplicar una técnica de preservación de reborde para facilitar a posteriori una buena colocación tridimensional del implante dental en sitios posteriores (39).

Otra variable en la preservación de reborde, es el cierre en primera o segunda intención, este tema parece ser controvertido en la literatura: mientras un metanálisis concluyó que el cierre primario tendía a minimizar la reducción del ancho de la cresta (11), otra revisión sugirió que no había diferencias estadísticamente significativas entre el cierre de la herida por primera o segunda intención (1) y en la revisión sistemática de Lee 2018 et al (31), sugirieron que la técnica sin colgajo fue más efectiva para preservar el ancho del reborde, la altura de hueso y el ancho de tejido queratinizado. En el presente estudio no encontró diferencias estadísticamente significativas entre las dos técnicas, tener en cuenta que se usaron biomateriales que cumplen los estándares científicos internacionales.

En la revisión sistemática y metanálisis de De Risi et al., mencionaron que los procedimientos de preservación de reborde son incapaces de acelerar o retrasar el proceso de curación fisiológica, simplemente porque no mejoran las modificaciones histológicas en sitios tratados, por ende no es necesario esperar más de 3 a 4 meses para la colocación del implante (35), por este motivo es que Guarnieri et al., (14), Barone et al., (15) y Walker et al., (16), emplearon 3 meses de seguimiento en sus respectivos estudios. Los demás ECAs $(9,17,19,21)$, emplearon 6 meses de seguimiento, además Lorenzetti et al., mencionó que las partículas de xenoinjerto mostraron una buena incorporación en el reborde recién formado a los 6 meses y garantizaron una densidad ósea suficiente para obtener una buena estabilidad primaria (37).

Es importante resaltar que en todos los estudios de la presente revisión sistemática se hace mención de la técnica exodoncia atraumática, que debe ir acompañado con técnica de preservación de reborde, con el objetivo de no provocar daños a la tabla óseas. En base a los resultados encontrados en la presente revisión y a las necesidades de tratamiento en otras situaciones clínicas, se propone el siguiente protocolo post extracciones de piezas posteriores que van a ser rehabilitados posteriormente con implantes dentales: - Evaluar la posibilidad de colocar un implante tipo 1 (inmediata post extracción) o tipo 2 (colocación temprana en tejidos blandos cicatrizados de 4 a 8 semanas) según Gallucci et al., (38)

- De no ser lo indicado se recomienda realizar una técnica de preservación de reborde.

- La colocación del implante tipo 1 ó 2 debe ser realizado por especialistas con alto nivel de experiencia y debe considerarse las siguientes variables: tablas óseas intactas, tabla ósea vestibular mayor a $1 \mathrm{~mm}$, ausencia de infección aguda en el sitio extracción, disponibilidad de hueso apical igual o mayor a $6 \mathrm{~mm}$ (cercanía al seno maxilar y conducto dentario inferior) y presencia de más de $2 \mathrm{~mm}$ de tejido queratinizado (36).

- La técnica de PRA inicia con una exodoncia mínimamente invasiva que incluya el uso de periótomos y separación de raíces (odontosección), siguiendo con el curetaje minucioso del alveolo, una vez que se tiene acceso visual del lecho alveolar se mide el ancho de la tabla vestibular mediante un calibrador quirúrgico y según el grosor tabla vestibular, se colocará el biomaterial, que según la evidencia disponible es el xenoinjerto.

- Es recomendable la colocación de material de relleno xenoinjerto principalmente en aquéllos casos 
de pared ósea delgada $<1 \mathrm{~mm}$ o dehiscencias. En casos de pared ósea vestibular gruesa $>1 \mathrm{~mm}$ se puede optar por el uso de membrana sin material de relleno. - Recomendamos un cierre en primera intención si existe adecuado fondo de surco y más de $2 \mathrm{~mm}$ de tejido queratinizado, en caso contrario y de realizarse esta técnica proponer una segunda intervención para la profundización de surco. También podemos optar por dejar la membrana expuesta (membranas de colágeno que cumplen los estándares científicos internacionales) de tal manera que la membrana se introduzca 2 a $3 \mathrm{~mm}$ dentro del margen gingival y se estabiliza con una sutura. El diagrama de flujo se muestra en la figura 2 .

Figura 2. Manejo clínico de una exodoncia en piezas molares

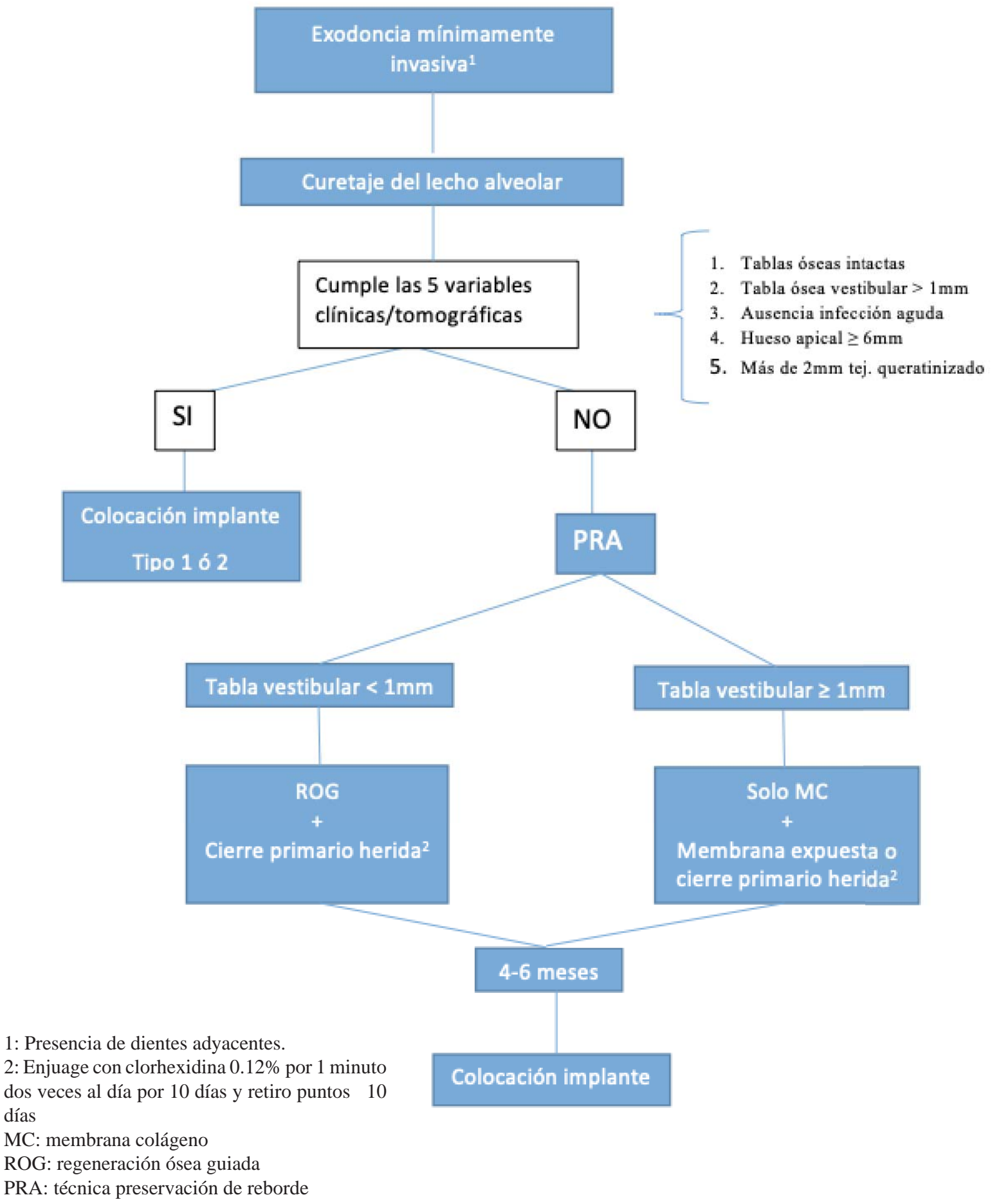




\section{Limitaciones de los artículos incluidos}

La identificación y localización de las piezas dentales intervenidas no fueron claras en algunos estudios (Premolares, molares), estas variaciones deberían ser consideradas para interpretar los resultados del presente estudio.El efecto de la técnica de preservación de reborde sobre los resultados reportados por el paciente (RRPs) no fueron considerados en la presente revisión, los RRPs son mecanismos fundamentales para calificar la experiencia del paciente en los resultados, además de auxiliar al clínico en puntos por mejorar en la intervención quirúrgica y el tamaño de la muestra fue reducida en algunos estudios.

\section{CONCLUSIONES}

La preservación de reborde alveolar en sitios posteriores conduce a mantener los tejidos duros y blandos existentes, reduce la neumatización sinusal y minimiza la reabsorción del hueso crestal generando un buen volumen de tejido óseo simplificando así los procedimientos para lograr una buena posición tridimensional del implante. Una buena técnica de exodoncia atraumática debe ser considerada para lograr mejores resultados en la técnica de preservación de reborde. Para tomar la decisión clínica de preservar o colocar implantes tipo 1 es importante definir cinco variables: tablas óseas intactas, la distancia del ápice al seno maxilar y al nervio dentario inferior, el grosor de la tabla ósea, la cantidad de tejido queratinizado presente y la ausencia de infección aguda en el sitio de extracción. Se sugiere implementar el mecanismo de medición: resultados reportados por el paciente (RRPs) en futuros ensayos clínicos aleatorizados para mejorar los resultados clínicos en los pacientes.

\section{Correspondencia}

Edwin Pascual Ramos Pilco

Correo electrónico: edy4208@gmail.com

\section{REFERENCIAS BILIOGRÁFICAS}

1. Tan WL, Wong TLT, Wong MCM, Lang NP. A systematic review of post-extractional alveolar hard and soft tissue dimensional changes in humans. Clin Oral Impl Res. 2012; 23 Suppl 5: 1-21.

2. Bassir SH, Alhareky M, Wangsrimongkol B, Jia Y, Karimbux N. Systematic review and Meta-Analysis of hard tissue outcomes of alveolar ridge preservation. Int
J Oral \& Maxillofac implants. 2018; 33(5): 979-994.

3. Araújo MG, Lindhe J. Dimensional ridge alterations following tooth extraction. An experimental study in the dog. J Clin Periodontol. 2005; 32: 212-218.

4. Hämmerle $\mathrm{CH}$, Araújo MG, Simion M, Osteology Consensus Group 2011. Evidence-based knowledge on the biology and treatment of extraction sockets. Clin Oral Implants Res. 2012; 23 Suppl 5: 80-82.

5. Hämmerle CHF, Cordaro L, Alccayhuaman KAA, et al. Biomechanical aspects: Summary and consensus statements of group 4. The 5th EAO consensus conference 2018. Clin Oral Implants Res. 2018; 18: 326-331.

6. Avila-Ortiz G, Chambrone L, Vignoletti F. Effect of alveolar ridge preservation interventions following tooth extraction: A systematic review and MetaAnalysis. J Clin Periodontol. 2019;46 Suppl 21:195223

7. Baumer D, Zuhr O, Rebele S, Hurzeler M. Socket Shield Technique for immediate implant placement clinical, radiographic and volumetric data after 5 years. Clin Oral Implants Res. 2017; 28: 1450-1458.

8. Darby I, Chen ST, Buser D. Ridge preservation techniques for implant therapy. Int J Oral Maxillofac Implants. 2009; 24: 260-271.

9. Jung RE, Sapata VM, Hämmerle CHF, Wu H, Hu $\mathrm{XL}$, Lin Y. Combined use of xenogeneic bone substitute material covered with a native bilayer collagen membrane for alveolar ridge preservation: A randomized controlled clinical trial. Clin Oral Implants Res. 2018; 29(5):522-529.

10. Marconcini S, Giammarinaro E, Derchi G, Alfonsi F, Covani U, Barone A. Clinical outcomes of implants placed in ridge-preserved versus nonpreserved sites: A 4-year randomized clinical trial. Clin Implant Dent Relat Res. 2018; 20(6):906-914.

11. Vignoletti F, Matesanz P, Rodrigo D, Figuero E, Martin C, Sanz M. Surgical protocols for ridge preservation after tooth extraction: A systematic review. Clinical Oral Implants Research. 2012; 23 Suppl 5: 22-38.

12. Moher D, Liberati A, Tetzlaff J, Altman DG. Preferred reporting items for systematic reviews and metaanalyses: The PRISMA statement. J Clin Epidemiol. 2009; 62(10): 1006- 1012.

13. Higgins JPT, Green S. Cochrane Handbook for Systematic Reviews of Interventions Versión 50-1. London: Cochrane collaboration; 2011 (citado el Agosto 2018). Disponible en http://handbook.cochrane. org

14. Guarnieri R, Stefanelli L, De Angelis F, Mencio F, Pompa G, Di Carlo S. Extraction socket preservation using porcine-derived collagen membrane alone or associated with porcine-derived bone. Clinical results of randomized controlled study. J Oral Maxillofac Res. 2017; 8(3): 5. 
15. Barone A, Toti P, Quaranta A, et al. Clinical and Histological changes after ridge preservation with two xenografts: preliminary results from a multicentre randomized controlled clinical trial. J Clin Periodontol. 2017; 44: 204-214.

16. Walker CJ, Prihoda TJ, Mealey BL, Lasho DJ, Noujeim M, Huynh-Ba G. Evaluation of healing at molar extraction sites with and without ridge preservation: A randomized controlled clinical trial. J Periodontol. 2017; 88(3): 241-249.

17. Lei C, Wu H, Lin Y, Di P, Chen B, Hu X. Application of xenograft for alveolar ridge preservation in posterior sites: a randomized controlled clinical trial. Zhonghua Kou Qiang Yi Xue Za Zhi. 2015; 50(9): 522-6.

18. Barone A, Ricci M, Tonelli P, Santini S, Covani $\mathrm{U}$. Tissue changes of extraction sockets in humans: a comparison of spontaneous healing vs. ridge preservation with secondary soft tissue healing. Clin Oral Implants Res. 2013;24(11):1231-7.

19. Festa VM, Addabbo F, Laino L, Femiano F, Rullo R. Porcine-derived xenograft combined with a soft cortical membrane versus extraction alone for implant site development: a clinical study in humans. Clin Implant Dent Relat Res. 2013;15(5):707-13.

20. Levi I, Halperin-Sternfeld M, Horwitz J, Zigdon-Giladi $\mathrm{H}$, Machtei EE. Dimensional changes of the maxillary sinus following tooth extraction in the posterior maxilla with and without socket preservation. Clin Implant Dent Relat Res. 2017;19(5):952-958.

21. Pang C, Ding Y, Hu K, Zhou H, Qin R, Hou R. Influence of preservation of the alveolar ridge on delayed implants after extraction of teeth with different defects in the buccal bone. Br J Oral Maxillofac Surg. 2016; 54(2): 176-80.

22. Sonick M, Abrahams J, Faiella RA. A comparison of the accuracy of periapical, panoramic and computerized tomographic radiographs in locating the mandibular canal. Int Oral Maxillofac Implants. 1994; 9: 455-460.

23. Kobayashi K, Shimoda S, Nakagawa Y, Yamamoto A. Accuracy in measurement of distance using limited CBCT. Int J Oral Maxillofac Implants. 2004; 19(2): 228-31.

24. Avila-Ortiz G, Elangovan S, Kramer KW, Blanchette D, Dawson DV. Effect of alveolar ridge preservation after tooth extraction: a systematic review and metaanalysis. J Dent Res. 2014; 93(10): 950-8.

25. Iocca O, Farcomeni A, Pardiñas Lopez S, Talib HS. Alveolar ridge preservation after tooth extraction: a Bayesian Network meta-analysis of grafting materials efficacy on prevention of bone height and width reduction. J Clin Periodontol. 2017; 44(1): 104-114.

26. Mardas N, Trullenque-Eriksson A, MacBeth N, Petrie A, Donos N. Does ridge preservation following tooth extraction improve implant treatment outcomes: a systematic review: Group 4: Therapeutic concepts \& methods. Clin Oral Implants Res. 2015; 26 Suppl 11: 180-201.

27. Vittorini Orgeas G, Clementini M, De Risi V, de Sanctis M. Surgical techniques for alveolar socket preservation: a systematic review. Int J Oral Maxillofac Implants. 2013; 28(4): 1049-61.

28. MacBeth N, Trullenque-Eriksson A, Donos N, Mardas $\mathrm{N}$. Hard and soft tissue changes following alveolar ridge preservation: a systematic review. Clin Oral Implants Res. 2017; 28(8): 982-1004.

29. Checchi V, Felice P, Zucchelli G, et al. Wide diameter immediate post-extractive implants vs delayed placement of normal-diameter implants in preserved sockets in the molar region: 1-year post-loading outcome of a randomised controlled trial. Eur J Oral Implantol. 2017; 10(3): 263-278.

30. Jung RE, Philipp A, Annen BM, et al. Radiographic evaluation of different techniques for ridge preservation after tooth extraction: a randomized controlled clinical trial. J Clin Periodontol. 2013; 40(1): 90-8.

31. Lee J, Lee JB, Koo KT, Seol YJ, Lee YM. Flap management in alveolar ridge preservation: A systematic review and meta-analysis. Int $\mathrm{J}$ Oral Maxillofac Implants. 2018; 33(3): 613-621.

32. Sharan A, Madjar D. Maxillary sinus pneumatization following extractions: a radiographic study. Int J Oral Maxillofac Implants. 2008; 23(1): 48-56.

33. Grusovin MG, Coulthard P, Worthington HV, George $\mathrm{P}$, Esposito $\mathrm{M}$. Interventions for replacing missing teeth: augmentation procedures of the maxillary sinus. London: Cochrane Database Syst Rev; 2010. (Citado en agosto del 2018) Disponible en: https://www. cochrane.org/CD003069/ORAL_interventions-forreplacing-missing-teeth-maintaining-and-recoveringsoft-tissue-health-around-dental-implants

34. Fan T, Li Y, Deng WW, Wu T, Zhang W. Short implants (5 to $8 \mathrm{~mm}$ ) versus longer implants $(>8 \mathrm{~mm}$ ) with sinus lifting in atrophic posterior maxilla: A meta-analysis of RCTs. Clin Implant Dent Relat Res. 2017; 19(1): 207215.

35. De Risi V, Clementini M, Vittorini G, Mannocci A, De Sanctis M. Alveolar ridge preservation techniques: a systematic review and meta-analysis of histological and histomorphometrical data. Clin Oral Implants Res. 2015; 26(1): 50-68.

36. Morton D, Gallucci G, Lin W, et al. Group 2 ITI Consensus Report: Prosthodontics and implant dentistry. Clin Oral Impl Res. 2018;29 Suppl 16:215223.

37. Lorenzetti M, Vono M, Lorenzetti V. A retrospective tomographic and histologic analysis of horizontal bone augmentation in maxillary atrophic ridges using resorbable membrane with anorganic bovine bonederived mineral and plasma rich in growth factors. Int $\mathrm{J}$ 
Periodontics Restorative Dent. 2019;39(3):399-406.

38. Gallucci G, Hamilton A, Zhou W, Buser D, Chen S. Implant placement and loading protocols in partially edentulous patients: A systematic review. Clin Oral Implants Res. 2018;29 Suppl 16:106-134.

39. Cha JK, Song YW, Park SH, Jung RE, Jung UW, Thoma DS. Alveolar ridge preservation in the posterior maxilla reduces vertical dimensional change: a randomized controlled clinical trial. Clin Oral Implants Res. 2019;30(6):515-523.

Recibido: 18-02-2019

Aceptado: 27-06-2019 